\title{
Osteotomien am Femurschaft und distalen Femur nach in Achsenabweichung verheilten Frakturen
}

\author{
J. M. Proske, A. Pommer, A. Dávid
}

\section{Zusammenfassung}

Eine posttraumatische Fehlstellung am Femurschaft kann nach konservativer Behandlung oder Marknagelung und seltener auch nach Plattenosteosynthese auftreten. Die Korrektur wird notwendig, wenn Achsenfehler funktionell nicht kompensiert werden können und eine Schädigung von Gelenken zu erwarten ist. Von Einfluss auf die Indikation und den Ort der Korrekturosteotomie sind die Beingeometrie, die muskuläre, ligamentäre und artikuläre Situation sowie die Weichteil- und Knochenqualität. Die Korrektur erfolgt nach Möglichkeit am Ort der Fehlstellung. Im Anschluss an die Osteotomie kann die Stabilisierung grundsätzlich mit einem Marknagel oder einer Plattenosteosynthese vorgenommen werden.

\section{Einleitung}

Die Kenntnis der physiologischen Beingeometrie ist Grundlage für Indikation und Planung von Korrekturosteotomien. Die mechanische Achse des Beines verläuft unter Normalbedingungen durch die Mitte des Femurkopfes, des Kniegelenkes und des oberen Sprunggelenkes. Die Femurschaftachse bildet mit der Längsachse der Tibia beim Erwachsenen den nach lateral offenen Tibiofemoralwinkel von $175^{\circ}$, gegen die Horizontale ist sie durch den Kniegelenksspalt um $82^{\circ}$ geneigt [4].

Bei den Achsenverhältnissen der Beine kann im Seitenvergleich eine Symmetrie

OP-JOURNAL 2000; 16: 57-60

(c) Georg Thieme Verlag Stuttgart · New York zugrunde gelegt werden. Hinsichtlich der Torsionen sind intraindividuelle Abweichung von bis zu $15^{\circ}$ am Oberschenkel physiologisch. Längentoleranzen betragen nach Untersuchung von Strecker et al. am Oberschenkel $1,2 \mathrm{~cm}$, am Unterschenkel $1,0 \mathrm{~cm}$ und für das gesamte Bein 1,4 cm [16].

Im Erwachsenenalter erworbene posttraumatische Längendifferenzen können funktionell nicht mehr voll kompensiert werden.

Posttraumatische Torsionsabweichungen werden am häufigsten nach Marknagelosteosynthesen beobachtet [3]. Durch die Erweiterung des Indikationsspektrums für Marknägel finden sich solche Fehlstellungen auch zunehmend metaphysär. Primäre Achsenfehlstellungen können nach Einschlagen eines Marknagels unter Verwindung durch elastische Rückstellkräfte auftreten. Bei fehlender kortikaler Abstützung und zu forcierter Belastungssteigerung resultieren sekundäre Achsenabweichungen oder Verkürzungen.

\section{Klinische und apparative Diagnostik}

Die klinische Untersuchung dient zunächst zur Diagnosesicherung, der Bewertung des Schweregrades einer Fehlstellung und der Beurteilung der Prognose des Patienten. Dabei muß insbesondere die aktive und passive Beweglichkeit des Bewegungsapparates erfasst werden.

Die Darstellung der Achsen in der Frontalund Sagittalebene erfolgt durch unter statischer Belastung im Seitenvergleich erstellter Röntgenaufnahmen. Für die Bestimmung von Torsionswinkeln und Längen ist die standardisierte CT-Untersuchung derzeitiger Goldstandard [17].

Insbesondere bei Kindern wird aufgrund der Bewegungsempfindlichkeit und der Strahlenbelastung durch die Computertomographie zunehmend ein dreidimen- sionales sonographisches Messsystem benutzt, das allerdings noch nicht routinemäßig eingesetzt wird [9]. Die MRTUntersuchung ist zwar günstig zur Beurteilung von Gelenkstrukturen, im Vergleich zu den anderen Untersuchungstechniken aber sehr kostenintensiv.

\section{Indikation zur Korrekturosteotomie}

Die Analyse von Fehlstellungen erfolgt zunächst in ihren Einzelkomponenten (Tab.1). Bei mehrdimensionalen Fehlstellungen entscheidet das Gesamtbild über die Indikation zur Korrekturosteotomie. Die im folgenden genannten Richtgrößen zur Korrektur monodimensionaler Fehlstellungen müssen daher bei komplexen Situationen relativiert werden. Die Toleranz für ein Genu valgum und ein Genu varum wird durch den möglichen Ausgleich im unteren Sprunggelenk bestimmt. Dieser ist für ein Genu valgum bis $10^{\circ}$ und für ein Genu varum bis $5^{\circ}$ möglich. Neben der knöchernen Geometrie ist immer die ligamentäre Stabilität mit zu beurteilen, da sich instabile Kollateralbänder durch eine additive Osteotomie straffen lassen. Eine Veränderung des Kniebasiswinkels oder eine Verdrehung des Kondylenblocks kann in der seitlichen Röntgenaufnahme bei Achsabweichungen in der Sagittalebene gezeigt werden. Die Indikationsgrenze liegt auf Grund der pathologischen Scherbela-

\begin{tabular}{lll}
$\begin{array}{lll}\text { Tab. } 1 \\
\text { scher Fehlstellungen }\end{array}$ & Tolen posttraumati- \\
\hline Dimension & Abweichung & $\begin{array}{l}\text { OP- } \\
\text { Indikation }\end{array}$ \\
\hline frontal & Varus & $5^{\circ}$ \\
& Valgus & $10^{\circ}$ \\
sagittal & Antekurvation & $15^{\circ}$ \\
& Rekurvation & $15^{\circ}$ \\
Drehung & Innendrehung & $15^{\circ}$ \\
& Außendrehung & $20-25^{\circ}$ \\
Länge & Verkürzung/ & $1,5 \mathrm{~cm}$ \\
& Verlängerung & \\
\hline
\end{tabular}


stung und der damit verbundenen Athrosegefahr bei einer Abweichung von $15^{\circ}$. Am distalen Femur tritt bei Antetorsionsfehlstellungen ein Streckdefizit im Kniegelenk schon bei geringerem Ausmaß auf. Ad-latus-Dislokationen beeinflussen die patellofemorale Geometrie. Ein Achsenversatz in der Frontalebene führt zu einer lateralen oder medialen patellofemoralen Hyperkompression. Die Indikation zur Umstellungsosteotomie orientiert sich dabei am klinischen Beschwerdebild des Patienten. Außenrotationsfehler sind ab 20-25 korrekturbedürftig, Innenrotationsfehler können bereits $a b$ $15^{\circ} \mathrm{zu}$ einer erheblichen Störung des Gangbildes führen. Eine posttraumatische Beinlängendifferenz von mehr als $1,5 \mathrm{~cm}$ sollte auf Grund kosmetischer und funktioneller Auswirkungen mit schmerzhaften Folgezuständen an Skelett und Weichteilen immer ausgeglichen werden. Neben der Analyse der Beinachsen kann die biomechanische Beanspruchung zur Beurteilung der subchondralen Knochendichte durch eine computertomographische Osteoabsorptiometrie bestimmt werden. Nach Wiederherstellung physiologischer Achsenverhältnisse kann dabei eine Änderung der Knochendichteverteilung beobachtet werden [10].

\section{Fehlstellungen im Kindesalter}

Posttraumatische Fehlstellungen im Kindesalter werden wegen der Möglichkeit zur Spontankorrektur in der Regel erst nach Wachstumsabschluss korrigiert.

Dabei zeigen kindliche Femurschaftfrakturen eine ausgesprochen günstige Neigung zur Spontankorrektur.

Verschiebungen um Schaftbreite, Frakturverkürzungen und Varus-/Valgusfehlstellungen von $15^{\circ}$ können ausgeglichen werden, wenn das Restwachstum noch 4 Jahre beträgt. Bei Kindern unter 10 Jahren werden auch Rotationsfehlstellungen durch Korrektur der Antetorsion auf der Gegenseite und spontane Außendrehung des Femurs distal der Fraktur am verletzten Bein korrigiert. Fehlstellungen die nach dem 10. Lebensjahr auftreten, werden kaum noch korrigiert. Eine Indikation zur Korrekturosteotomie vor Abschluss des Längenwachstums besteht bei einer auf Dauer nicht tolerablen Fehlbelastung benachbarter Gelenke auf Grund einer ausbleibenden Spontankorrektur oder wenn nach weiterem progredienten Fehlwachstum technische Operationsschwierigkeiten zu erwarten sind.

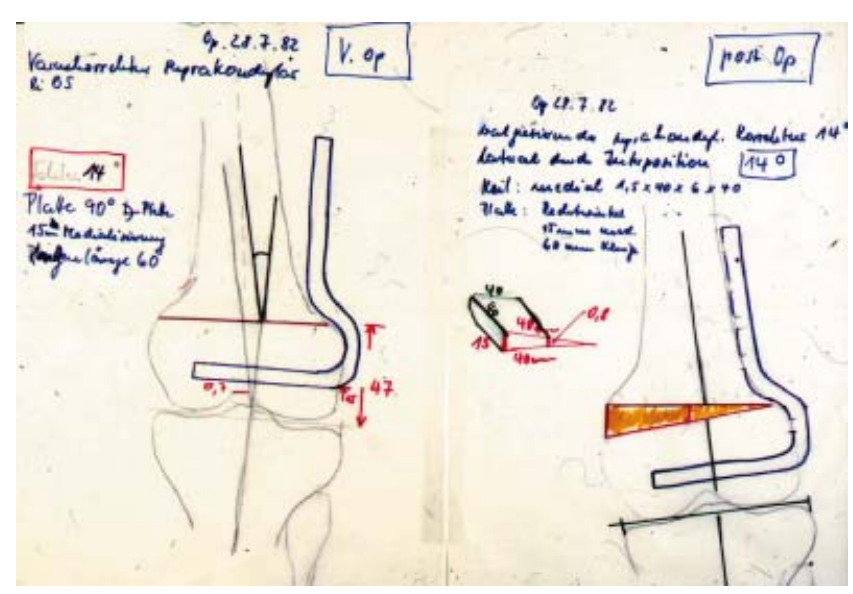

Abb. 1 Beispiel einer präoperativen Planungsskizze.

\section{Operationsplanung}

Die operative Planungsskizze erfolgt an der Ganzbeinaufnahme in der abgebildeten Fehlstellungsebene. Dabei kann die Ausrichtung der Fehlstellungsebene graphisch oder mathematisch lokalisiert und quantifiziert werden (Abb.1). Ist eine Deformität im a.-p.- und Seitbild sichtbar und besteht zusätzlich eine Torsionsfehlstellung muss die Korrekturebene über ein Vektordiagramm ermittelt werden. Die Ausgradung der Extremität ergibt sich aus der Korrektur um die hierbei ermittelten Drehpunkte. Je weiter der Drehpunkt in der Konvexität liegt, desto größer ist der Verlängerungseffekt. Bei einem Drehpunkt in der Konkavität bewirkt die Korrektur eine Verkürzung. Das Ziel einer Korrekturosteotomie ist die Wiederherstellung des anatomischen Achsenzustandes der Extremität.

Eine Osteotomie wird in Höhe der Fehlstellung angestrebt.

Eine Überkorrektur von $2-4^{\circ}$ wird nur dann durchgeführt, wenn eine mediale oder laterale Gonathrose vorliegt oder droht, wie etwa bei Knorpeldestruktion einer Kondyle.

\section{Operationstechnik}

Die meisten Korrekturosteotomien an den unteren Extremitäten werden aufgrund von Fehlstellungen in der Frontalebene durchgeführt. Kongenitale oder posttraumatische Achsenabweichungen werden dabei operationstechnisch gleich behandelt.

Für die Stabilisierung nach einer Korrekturosteotomie stehen grundsätzlich interne und externe Techniken zur Verfügung.
Die Anwendung interner Verfahren wird durch ihr Stabilisierungspotential, die verfügbare Knochensubstanz und den Weichteilstatus limitiert. Externe Verfahren werden bei Deformitäten in unmittelbarer Gelenknähe, bei größerem Längenausgleich sowie sehr schlechten Weichteil- und Vaskularitätsverhältnissen eingesetzt.

\section{Technik der Osteotomie}

Es werden grundsätzlich kleine Zugänge mit möglichst geringer Weichteilmobilisierung vom Knochen angewandt. Der Knochen sollte nicht zirkulär freigelegt und der Weichteilmantel nur soweit wie nötig reseziert werden. In der supracondylären Region sind zirkulär positionierte Hohmann-Hebel zum Schutz der Gefäß-Nervenbahnen erforderlich. Im Femurschaftbereich sind diese entbehrlich. Nach Dekortikation der Osteotomieregion wird zur Optimierung des Heilungsverlaufes bei der Durchtrennung des Knochens nach Möglichkeit keine oszillierende Säge sondern ein scharfer Meißel verwendet [1].

\section{Schaftkorrekturen}

Einzeitige Längenkorrekturen sind am Oberschenkel in ihrem Ausmaß durch die Dehnungskapazität der Weichteile auf $3 \mathrm{~cm}$ begrenzt. Durch einseitige Verkürzung und gegenseitige Verlängerung könnten so bis zu $6 \mathrm{~cm}$ ausgeglichen werden. Verkürzungen lassen sich durch eine quere Osteotomie im metaphysären Bereich und nachfolgende Winkelplattenosteosynthese durchführen. Verlängerungen können im metaphysären Bereich einzeitig durch treppenförmige Osteotomie und Stabilisierung mittels Winkelplattenosteosynthese erreicht werden [11]. Indikationen für Distraktionsverfahren sind Beinlängendifferenzen von mehr als $3 \mathrm{~cm}$ sowie einfache und komplexe Achsenfehlstellungen. 
Monolaterale Fixateure werden zur isolierten Verlängerung von Ober- und Unterschenkel eingesetzt, während zur Achsenkorrektur insbesondere in mehreren Ebenen Ringfixateure aufgrund ihrer Komplexität besser geeignet sind [7].

Die Empfehlung hinsichtlich des maximal anzustrebenden Längenausgleichs beträgt für das Femur 6-10 cm. Achsenkorrekturen sind sehr genau durchführbar, da die den individuellen Deformitäten zugrundeliegenden Krümmungszentren berücksichtigt werden können und durch eine gezielte Versetzung von Korrekturachsen eine Translation ermöglicht wird. Die alleinige Distraktion mit Hilfe von lateralen Monofixateuren kann zu Varusfehlstellungen führen, da die medialen Weichteile nicht im gleichen Maße distrahiert werden wie die lateralen. Dieser Nachteil wird durch gleichzeitige Platzierung eines unaufgebohrten Marknagels mit dünnem Durchmesser zum Teil kompensiert. Dabei erfolgt die Verriegelung zunächst proximal. Nach Erreichen der angestrebten Beinlänge wird der Marknagel distal verriegelt und der Fixateur entfernt. Die Liegezeit des Fixateur-externe kann dadurch um ca. 60\% reduziert, eine Refraktur verhindert und die Behandlungsdauer verkürzt werden [12].

Bei liegendem Marknagel und isoliertem Drehfehler ist eine Torsionskorrektur über dem Marknagel möglich.

Die distalen Verriegelungsschrauben werden dabei gelöst und nach Korrektur der Fehlstellung neu platziert (Abb.2). Dabei sollte eine Torsionssicherung durch eine Plattenosteosynthese erfolgen, da eine erneute Verriegelung den Rückstellkräften der Fragmente nicht immer standhält [15]. Bei Neuimplantation eines Marknagels zu Korrektur von Varus-/Valgusfehlstellungen kann hingegen auf die ergänzende Platte verzichtet werden (Abb.3). Betz et al. entwickelten einen voll implantierbaren programmierbaren Distraktionsnagel. Eine äußere Distraktionsosteosynthese wird dabei nicht mehr notwendig [2]. Diese recht teuren Systeme befinden sich derzeit in klinischer Erprobung.

\section{Korrekturen am distalen Femur}

Je nach frontaler Neigung des Tibiaplateaus und der Knorpelqualität im Kniegelenk erfolgt bei mehrdimensionalen Fehlstellungen die Osteotomie supracondylär oder im Tibiakopfbereich. Unter Berück-
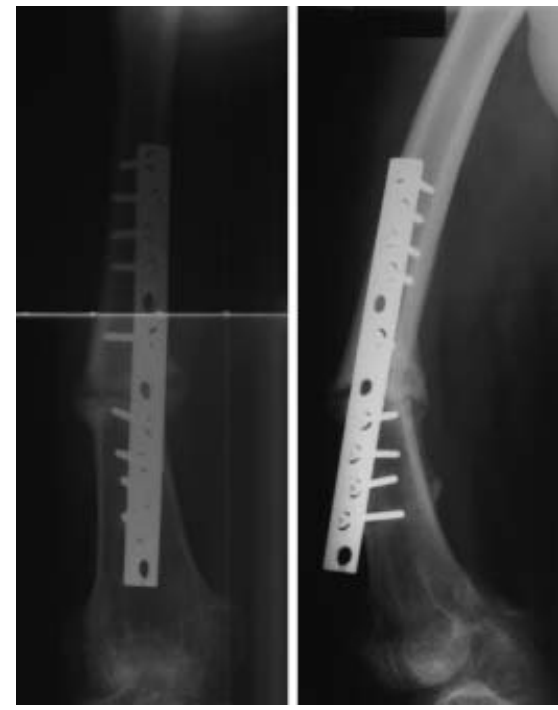

Abb. 2a In Innnenrotationsfehlstellung verheilter Oberschenkelschaftbruch.
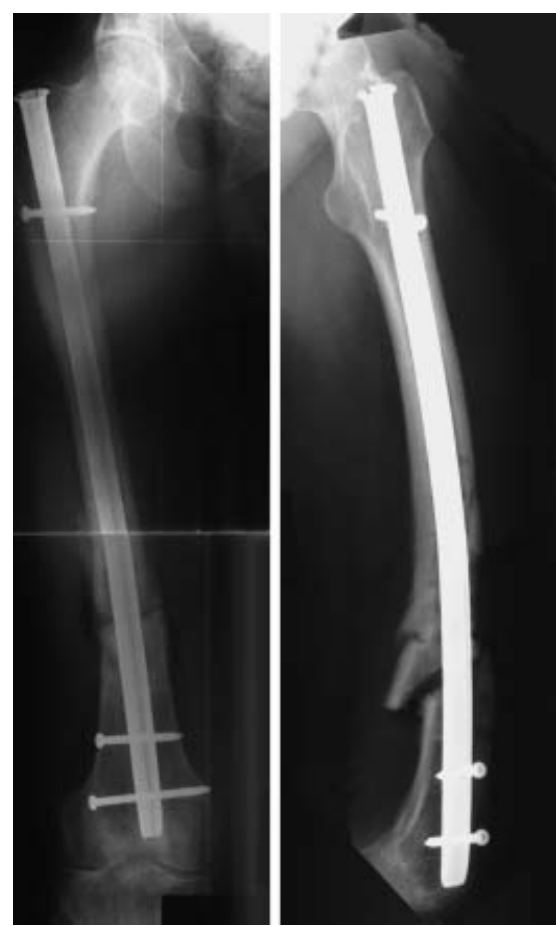

Abb.2b Achsenkorrektur über eingebrachten Marknagel und Spongiosaplastik.

sichtigung der Längenverhältnisse kann sie subtraktiv oder additiv durch Einbringen eines auto- oder allogenen Knochenspans erfolgen. Der Korrekturwinkel orientiert sich am Ausmaß der gewünschten Korrektur und der festgelegten Osteotomiehöhe. Bei gleichzeitiger Verkürzungs- und Torsionskorrektur sollte nicht mehr als $3,5 \mathrm{~cm}$ verkürzt und $45^{\circ}$ gedreht werden, da andernfalls eine Fehlbelastung des Kniegelenks oder Insuffizienz der Quadrizepsmusku-
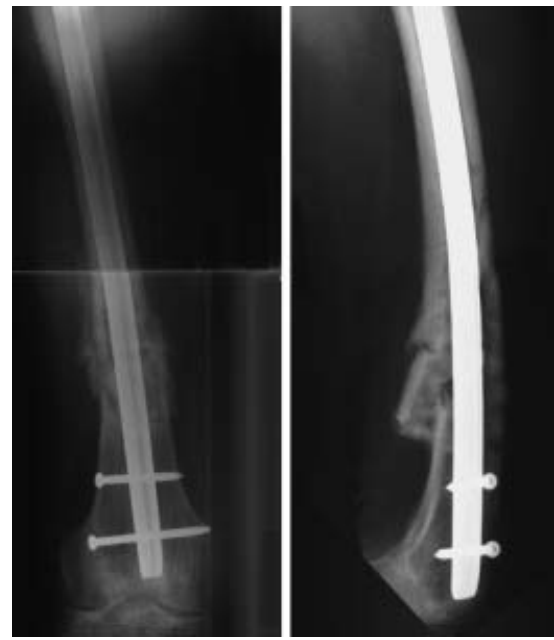

Abb.2c Ausheilungsergebnis nach 3 Monaten.

latur als Komplikation resultieren können.

Die Stabilisierung einer queren suprakondylären Korrekturosteotomie erfolgt bevorzugt mit Winkelplatten (Abb. 4).

Diese können prinzipiell lateral oder medial platziert werden. Sie werden in konventioneller Technik mit Ausnutzung exzentrischer Bohrungen und eines Plattenspanngerätes montiert. Eine Zugschraube wird dabei zusätzlich platziert. Bei Bandinsuffizien am Kniegelenk sind additive Techniken zu bevorzugen [8,13]. Sagittale Achsabweichungen werden analog zu Korrekturen in der Frontalebene additiv oder subtraktiv extendiert oder flektiert (Abb.5). Ad-latus-Dislokationen werden im allgemeinen durch eine quere Osteotomie ausgeführt. Bei gleichzeitiger Längenkorrektur bieten sich gelegentlich auch schräge Osteotomien an. Eine weichteilschonende Operationstechnik mit Erzielung übungsstabiler Osteosynthesen und adäquater Nachbehandlung ist Vorraussetzung für eine Operation in der Nähe zum Kniegelenk.

\section{Postoperative Komplikationen}

Wichtige planerische und operationstechnische Fehler bei allen genannten Verfahren sind eine falsche Osteotomiehöhe, eine intraartikuläre Metallage sowie Dehnungsschäden an nervalen Strukturen und am Gelenkknorpel [14]. Postoperativ auftretene Komplikationen sind ein Kompartmentsyndrom insbesondere bei ausgedehnter Devastierung der Weichteile und erzwungenem Faszienverschluss. Neben den genannten Faktoren führt ein Verfahrenswechsel von 

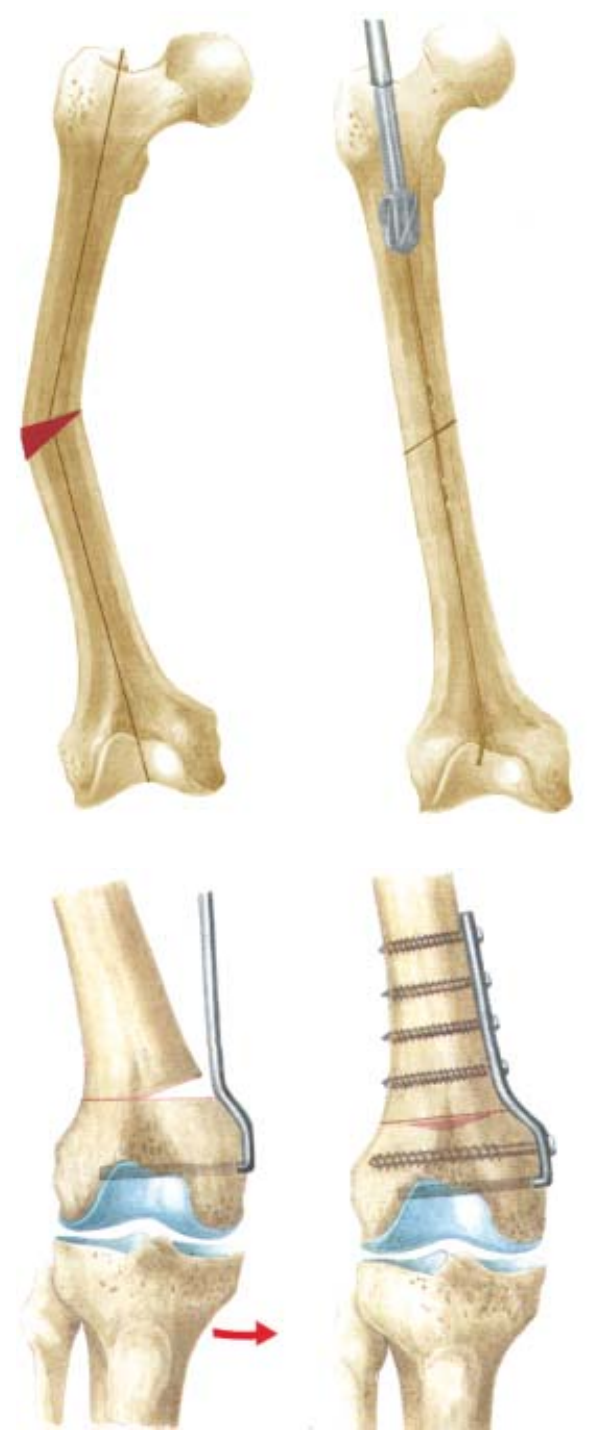

Abb. 4 Supracondyläre Korrekturosteotomie mittels einer medialen Winkelplatte.

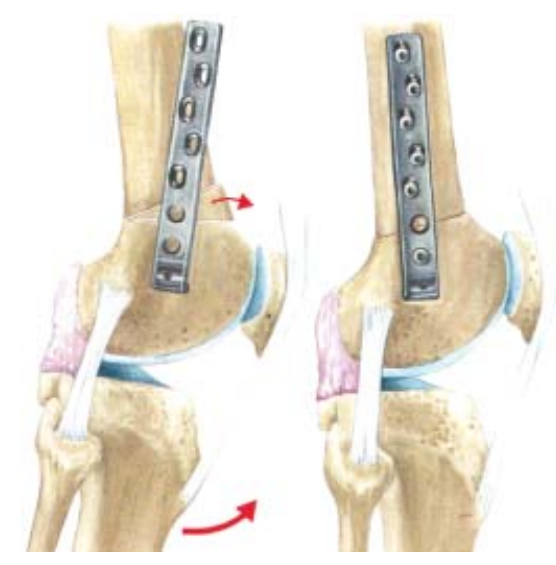

Abb. 5 Supracondyläre, extendierende Korrekturosteosynthese.

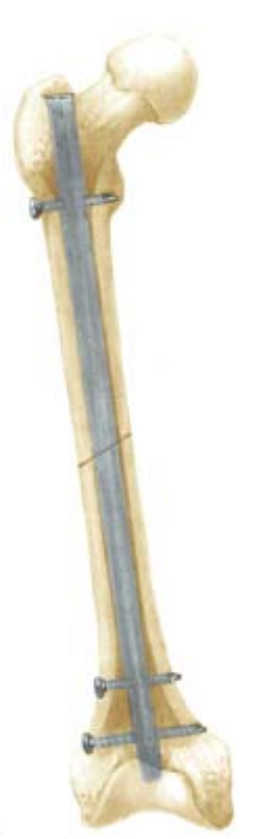

Abb. 3 Einbringen und Verriegelung eines Marknagels nach Varuskorrektur.

einer externen auf eine interne Osteosynthese zu einer Erhöhung des Infektionsrisikos. Schließlich kann es nach einer additiven Osteotomie zu einer Spanresorption kommen $[5,6]$.

\section{Schlussfolgerung}

Bei pathologischen Fehlstellungen an den unteren Extremitäten ermöglicht die Wiederherstellung der mechanischen Achsverhälnisse die Prävention einer Gelenkdegeneration und Rekonstruktion einer belastungsfähigen Extremität. Nach subtiler Diagnostik und Operationsplanung stehen mit dem Fixateur externe, der Marknagelung und der Plattenosteosynthese unter den jeweiligen anatomischen Vorraussetzungen differenzierte Systeme zur Versorgung zur Verfügung. Verlängerungsosteotomien werden dabei zunehmend in der Distraktionstechnik nach Ilisarov vorgenommen.

\section{Literatur}

${ }^{1}$ Baumgart R, Kettler M, Zeiler C, Betz A, Schweiberer L. Möglichkeiten der Knochendurchtrennung. Osteotomie- und Kortikotomietechniken. Unfallchirurg 1997; 100: $797-804$

${ }^{2}$ Betz A, Baumgart R. Erstes voll implantierbares intramedulläres System zur Kallusdistraktion-Marknagel mit programmierbarem Antrieb zur Beinverlängerung und Segmentverschiebung. Chirurg 1990; 61: 605-609

${ }^{3}$ Braten M, Terjesen T, Rossvoll I. Femoral shaft fractures treated by intramedullary nailing. A follow-up study focusing on problems related to the method. Injury 1995; 56: $379-383$

${ }^{4}$ Debrunner AM. Der Bewegungsapparat: Biomechanik und Pathophysiologie. In: Debrunner AM (Hrsg.): Orthopädie, orthopädi- sche Chirurgie. Hans Huber Verlag Bern 1995.

${ }^{5}$ Herron LD, Amstutz HC, Sakai DN. One stage femoral lengthening in the adult. Clin Orthop 1978; 136: 74-82

${ }^{6}$ Höntzsch D, Weller S, Engels C, Kaiserauer S. Die Verfahrenswechsel vom Fixateur externe zur Marknagelosteosynthese an $\mathrm{Fe}$ mur und Tibia. Aktuelle Taumatol 1993; 23: $21-35$

7 Ilizarov GA. Clinical application of the tension-stress effect for limb lengthening. Clin Orthop 1990; 250: 8-26

${ }^{8}$ Knapp DR, Price CT. Correction of distal femoral deformity. Clin Orthop 1990; 255: 75-80

${ }^{9}$ Keppler P, Strecker W, Kinzl L, Simmnacher $\mathrm{M}$, Claes L. Die sonographische Bestimmung der Beingeometrie. Orthopäde 1999; 28 : 1015-1022

${ }^{10}$ Müller-Gerbl M, Griebl A, Putz R, Träger KH, Goldmann A, Kuhr M. Die Reaktion des subchondralen Knochens auf Achsenfehlstellungen des Kniegelenkes und deren Korrektur durch Osteotomien. In : Strecker (Hrsg.): Posttraumatische Beindeformitäten. Analyse und Korrektur. Springer-Verlag Berlin 1997; $97-100$

${ }^{11}$ Murray DW, Kambouroglou G, Kenwright J. One-stage lengthening for femoral shortening with associated deformity. J Bone Joint Surg Br 1993; 4: 566-571

${ }^{12}$ Paley D, Herzenberg JE, Paremain G, Bhave A. Femoral lengthening over an intramedullary nail. A matched-case comparison with Ilizarov femoral lenghtening. J Bone Joint Surg Am 1997; 79: 1464-80

13 Payne LZ, DeLuca PA. Intertrochanteric versus supracondylar osteotomy for severe femoral anteversion. J Pediatric Orthop 1994; 14: 39-44

${ }^{14}$ Stanitski DF, Rossman K, Toroisian M. The effect of femoral lengthening on knee articular cartilage: the role of apparatus extension across the jomt. J Pediatr Orthop 1996; 16 : $151-154$

${ }^{15}$ Strecker W, Hoellen I, Keppler P, Suger G, Kinzl L. Torsionskorrekturen nach Marknagelosteosynthesen der unteren Extremität. Unfallchirurg 1997; 100: 29-38

${ }^{16}$ Strecker W, Keppler P, Franzreb M, Keck S, Kinzl L. Längen und Torsionen der unteren Extremitäten. In : Strecker (Hrsg.): Posttraumatische Beindeformitäten. Analyse und Korrektur. Springer-Verlag Berlin 1997; $75-86$

${ }^{17}$ Waidelich H-A, Strecker W, Schneider E. Computertomographische Torsionswinkel und Längenbestimmung an der unteren Extremität. Fortschr Röntgenstr 1992; 157: 245-251

Dr. med. J. M. Proske

Assistenzarzt

A. Pommer

Leitender Oberarzt

Prof. Dr. med. A. Dávid

Direktor

Klinik für Unfall- und Wieder-

herstellungschirurgie

Klinikum Wuppertal

Heusnerstraße 40

42883 Wuppertal 